Prof. Dr. Peter Berlit

Deutsche Gesellschaft für Neurologie (DGN)

Reinhardtstr. 27C, 10117 Berlin, Deutschland

berlit@dgn.org

\title{
Aus- und Weiterbildung - auch in Zeiten der Coronaviruspandemie
}

In den Zeiten der Coronaviruspandemie ist es gar nicht so einfach, sich mit Themen wie Aus- und Weiterbildung zu befassen. Aber gerade diese weltweite Krisensituation zeigt uns ja, wie wichtig gut ausgebildete Ärztinnen und Ärzte sind. Und wie bedeutend es ist, sich auch in Quarantäne fort- und weiterbilden $\mathrm{zu}$ können. Dieses Editorial schreibe ich aus dem Homeoffice, während draußen das kulturelle und gesellschaftliche Leben praktisch zum Erliegen gekommen ist.

Unser multimediales Projekt RE imagine MEDICINE ergab, ebenso wie die Umfragen bei unseren jungen Neurologinnen und Neurologen, Niedergelassenen und Weiterbildungsassistentinnen und -assistenten, dass die Themen Aus- und Weiterbildung für unsere Mitglieder von allergrößter Bedeutung sind. Der Wunsch, dabei möglichst das bestehende Wissen auf dem neuesten Stand jederzeit verfügbar zu haben, am besten auf dem Handy oder Tablet, ist gerade in Zeiten der aktuellen Krise absolut verständlich.

Es ist zweifelsohne wertvoll, dass die Inhalte vieler Beiträge unserer Jahrestagung, insbesondere der Fortbildungsakademie, digital so angeboten werden, dass zu Hause nachgelernt werden kann - und dabei sogar noch CME-Punkte erworben werden können! Auch unsere Mitgliederzeitschrift steht mit ZOOM (neurologische Krankheitsbilder auf einen Blick [Rubrik]), SOP („standard operating procedures“), Scientific News und den anderen Ihnen bekannten praxisrelevanten Rubriken in digitaler Form zur Verfügung. Fallorientiertes Lernen ist in Aus- und Weiterbildung besonders effektiv, und diesen Bereich wollen wir mit Lernfällen für die Facharztprüfung kontinuierlich weiter ausbauen.

Was sich aber unsere Assistentinnen und Assistenten ebenso wie die niedergelassenen Kolleginnen und Kollegen ebenfalls wünschen, sind praktische Handlungsanweisungen - möglichst unter Miteinbeziehung von Videos und Audiodateien. Unsere Assistentinnen und Assistenten benötigen unbedingt die relevanten Inhalte für die Vorbereitung zur Facharztprüfung. In der Praxis und am Krankenbett sind Handlungshilfen für rasche Ent-

DGNeurologie 2020 • 3 (3): 183-184

https://doi.org/10.1007/s42451-020-00182-w

๑) Springer Medizin Verlag GmbH, ein Teil von Springer Nature 2020 scheidungen, aber auch Inhalte zur Vertiefung und Auffrischung des Wissens gefragt. Dabei müssen natürlich die Inhalte der neuen Weiterbildungsordnung ebenso wie unsere Leitlinien berücksichtigt werden.

Wenn, wie von politischer Seite gewünscht, die Aus- und Weiterbildung zunehmend auch im ambulanten Sektor stattfinden soll, werden digitale unterstützende Angebote zunehmend wichtiger. Dies gilt insbesondere für die Bereiche, in denen Handlungskompetenz gefordert ist, beispielsweise die Schlaganfallund Notfallversorgung, aber auch für die Neuroimmunologie und die seltenen Erkrankungen, von denen 80 \% mit neurologischen Symptomen einhergehen. So müssen die Spezialseminare und Hands-on-Kurse auf unserer Jahrestagung und bei den regelmäßigen Fortbildungsveranstaltungen der DGN (Deutsche Gesellschaft für Neurologie) zunehmend ausgebaut werden, auch mit digitalen Angeboten und entsprechendem Videounterrichtsmaterial.

Aber wir wollen auch schon Studierende für das Fach Neurologie begeistern. Der Entwurf der neuen Approbationsordnung für Ärztinnen und Ärzte sieht eine Aufwertung im Bereich der Allgemeinmedizin und der Ausbildung im ambulanten Bereich vor. Es ist sicher gut, dass eine höhere Wissenschaftlichkeit mit einem longitudinalen Track in der Ausbildung angestrebt wird, und die DGN begrüßt auch, dass im praktischen Jahr (PJ) mit der ganzheitlichen Betreuung von mindestens 2 Patientinnen bzw. Patienten von der Aufnahme bis zur Entlassung eine Aufwertung der praktischen, auch interprofessionellen, Ausbildung vorgesehen ist. Leider führt die vorgesehene Aufwertung der Allgemeinmedizin zu einer Reduzierung der Spezialdisziplinen allgemein, und der Neurofächer im Besonderen. Wir glauben, dass ein neurologisches Blockpraktikum innerhalb der studentischen Ausbildung zwingend erforderlich ist, um die Relevanz neurologischer Erkrankungen adäquat abzubilden. Neurologische Krankheitsbilder sind in der interdisziplinären Notaufnahme am dritthäufigsten, und die Prävalenz neurodegenerativer Erkrankungen steigt mit der Alterspyramide. In der neurologischen Ausbildung sollten auch die Partnerfächer Neurochirurgie, Neuroradiologie, Neurorehabilitation und Neurogenetik adäquat abgebildet sein. Und die Immunologie als wichtiges Querschnittsfach ist aus neurologischer Sicht unbedingt zu berücksichtigen. Das Staatsexamen sollte im Sinne der Schwerpunktbildung grundsätzlich eine 
praktische klinische Prüfung an der Patientin/am Patienten auch im Wahlfach beinhalten.

Sowohl bei den Themen der Ausbildung im Entwurf der neuen Approbationsordnung als auch bei den Inhalten der Weiterbildung in der neuen Weiterbildungsordnung stehen wir im engen Diskurs mit den Entscheidungsträgern, um zu erreichen, dass unser Fach adäquat abgebildet wird. Und gemeinsam mit unserer neuen Kommission Lehre werden wir neue Formate und Inhalte der Wissensvermittlung entwickeln. Wenn Sie hierzu Anregungen haben, lassen Sie es mich wissen - und: bleiben Sie gesund!

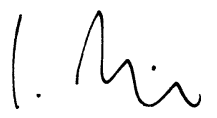

Ihr Peter Berlit

Interessenkonflikt. P. Berlit gibt an, dass kein Interessenkonflikt besteht.

\section{Aktuelle Entwicklung zu COVID-19}

Auf der Homepage SpringerMedizin.de finden Sie ein immer aktuelles Dossier mit Beiträgen, Forschungsarbeiten und Ergebnissen zu SARS-CoV-2 sowie relevanten Links.

SpringerNature.com/de verlinkt auf eine eigene Landingpage mit einer Vielzahl an Information sowie freiem Zugriff auf die COVID-19-Contentplatformen von Nature Research, BioMed Central (BMC) und Springer. 